\title{
Intervenciones breves: una oportunidad para reducir el consumo excesivo de alcohol entre los jóvenes*
}

\author{
Heather, N.*; Kaner, E.** \\ (*) Centre for Alcohol \& Drug Studies. Newcastle City Health NHS Trust \& University of Northumbria at Newcastle, UK \\ $\left.{ }^{* *}\right)$ Department of Primary Health Care, University of Newcastle upon Tyne, UK \\ Enviar correspondencia a: \\ Nick Heather. Centre for Alcohol \& Drug Studies. Plummer Court, Carliol Place, Newcastle upon Thyne. NE1 6UR, UK. Tel: +44(0)191 219 5648; \\ e-mail: nheather.newcads@onyxnet.co.uk
}

\section{RESUMEN}

Tras una serie de investigaciones, las intervenciones breves contra el consumo excesivo de alcohol en algunos marcos de la asistencia sanitaria han demostrado ser eficaces a la hora de reducir el consumo entre aquellos que abusan del alcohol. La puesta en práctica general y rutinaria de intervenciones breves en dichos marcos resultarían claramente ventajosas para la salud pública. El presente artículo examina datos que prueban la eficacia de las intervenciones breves en una serie de marcos médicos. Reciben especial atención los estudios WHO sobre "Detección y Control de los Problemas relacionados con el Alcohol en la Asistencia Sanitaria Primaria", incluyendo la actual fase IV de ese proyecto, relacionada con las estrategias de desarrollo para la puesta en práctica de la identificación temprana y de la intervención breve en el consumo excesivo de alcohol dentro de los sistemas de asistencia sanitaria primaria de los países participantes. El artículo se centra también en el potencial de las intervenciones breves para reducir los problemas ligados al alcohol, concretamente entre los jóvenes. Se analiza la pertinencia de ese objetivo en una serie de marcos de la asistencia sanitaria (en el ejercicio de la medicina general, entre los pacientes ingresados en un hospital, en los departamentos de urgencia). Se discuten, asimismo, algunos de los resultados obtenidos al tomar en consideración las intervenciones breves en el campo del alcohol entre los jóvenes. El artículo finaliza con una serie de conclusiones referentes al estudio y a la política de intervenciones breves en general y de intervenciones breves adaptadas a las necesidades de los jóvenes.

Palabras clave: Consumo abusivo de alcohol/ Daños relacionados con el alcohol/ Intervenciones breves/ Marcos médicos/ Jóvenes/ Borracheras.

\section{SUMMARY}

Brief interventions against excessive alcohol consumption delivered in health care settings have been shown by research to be effective in reducing consumption among excessive drinkers. The widespread and routine implementation of brief interventions in these settings would lead to substantial gains for public health. This paper reviews evidence for the effectiveness of brief interventions in a range of medical settings. Special mention is made of the WHO series of studies on "The Detection and Management of Alcohol-related Problems in Primary Health Care", including the current Phase IV of this project concerned with developing strategies for implementing early identification and brief intervention for excessive drinking in the primary health care systems of participating countries. Attention then turns to the potential of brief interventions for reducing alcohol problems specifically among young people. The relevance to this aim of a range of health care settings -general medical practice, general hospital wards, accident and emergency departments- is reviewed. Particular issues that arise when considering brief alcohol interventions for young people are discussed. The paper ends with a series of conclusions regarding research and policy for brief interventions in general and brief interventions adapted for the needs of young people.

Key words: Excessive drinking/ Alcohol-related harm/ Brief interventions/ Medical settings/ Young people/ Binge drinking.
$\mathbf{L}$ as intervenciones breves llevadas a cabo en algunos marcos de la asistencia sanitaria han demostrado ser eficaces, según las investigaciones, a la hora de reducir el consumo entre los individuos que abusan del alcohol (bebedores de riesgo y bebedores a los que el alcohol resulta ya nocivo) $)^{1-5}$. Asimismo, resulta ostensible que una puesta en práctica general y rutinaria de las intervenciones breves en dichos mar-

"El presente artículo se ha basado en una presentación a un grupo de trabajo sobre Sistemas sanitarios y Alcohol en la Conferencia Ministerial sobre Jóvenes y Alcohol, Estocolmo, Suecia, 19-23 de febrero, 2001. 
cos resultarían claramente ventajosas para la salud pública $^{6,7}$. Por desgracia, todo parece indicar que los profesionales de la asistencia sanitaria han sido muy lentos a la hora de incorporar a su práctica habitual las intervenciones breves en el campo del alcohol, por lo que es necesario realizar un esfuerzo mayor a fin de persuadir a doctores, enfermeras y demás personal sanitario pertinente de la importancia de su aplicación?.

Al hablar de intervenciones breves nos referimos a una categoría muy amplia de actividades cuyo rasgo común es ser llevadas a cabo por médicos y demás trabajadores sanitarios como parte habitual de su trabajo. Las intervenciones breves engloban desde una información de 5 a 10 minutos o una consulta con un médico de familia, hasta 2-3 sesiones de entrevistas motivacionales $u$ otra forma de asesoramiento ofrecido dentro de un hospital general por una enfermera especialmente preparada para ello. En todos los casos, sin embargo, es característico que la intervención sea más breve que el tratamiento ofrecido en casos de adicción al alcohol por organismos especializados. Las intervenciones breves son "oportunistas", en el sentido de que sus destinatarios todavía no se han quejado de tener un problema con el alcohol, pero han sido identificados a través de un chequeo o de cualquier otro medio como individuos que consumen alcohol a niveles que comportan riesgo o daño tanto para su salud como para su bienestar. Dichas intervenciones se efectúan en parte con el fin de lograr una identificación temprana y una prevención secundaria de problemas graves con el alcohol, así como para contribuir al funcionamiento de las medidas tomadas por la sanidad pública para reducir la alta prevalencia de bebedores de riesgo y de bebedores a los que el alcohol resulta nocivo, y que encontramos hoy en día en casi todas las sociedades. Las intervenciones breves no van dirigidas a bebedores problemáticos con niveles muy severos de dependencia y con problemas derivados de dicho consumo, quienes necesitan tratamientos más intensivos en centros especializados ${ }^{8}$

El presente artículo analizará en primer lugar los datos que nos permiten ver la eficacia de las intervenciones breves en general. Centraremos después nuestra atención en el potencial de las intervenciones breves a la hora de reducir los problemas ligados al alcohol, concretamente entre los jóvenes.

\section{Eficacia de las intervenciones breves en el marco de la asistencia sanitaria}

\section{Asistencia primaria sanitaria}

La eficacia de las intervenciones breves es más manifiesta en el marco de la asistencia sanitaria primaria. Algunas pruebas controladas aleatorias (RCTs) realizadas en distintos lugares del mundo han hecho ostensible que los individuos que han recibido la ayuda de intervenciones breves, de manos de médicos de familia 6 ,9-14, "consejeros de salud" 15 o equipos de médicos y enfermeras ${ }^{16-17}$, muestran mayor reducción en el consumo como consecuencia de ello que los grupos de control. Entre las mujeres, dos estudios han intentado demostrar sin conseguirlo las ventajas de la intervención, ya que los grupos de control también han mostrado reducciones significativas en el consumo ${ }^{15,18}$. Todo parece indicar que, en dichos estudios, la propia evaluación actuó como una forma de intervención entre las mujeres, despertando en ellas cierta inquietud sobre el nivel de consumo e incrementando su buena disposición a cambiar el comportamiento ante la bebida; en un estudio cuyo propósito de evaluación se "ocultaba" a quienes participaban en él ${ }^{12}$, las mujeres que consumían alcohol de forma abusiva mostraron un efecto ante la intervención al menos tan marcado como los hombres.

Mientras los estudios anteriormente citados hacían un seguimiento de los pacientes que no sobrepasaba el año, una investigación reciente de Fleming y colaboradores en Wisconsin ${ }^{19}$ afirmó que, tras las intervenciones breves, el consumo se reducía durante un período de seguimiento de cuatro años. Se hallaron, por otra parte, importantes beneficios económicos derivados de las intervenciones breves ${ }^{19,20}$, estimándose un ahorro en los costes futuros de la sanidad pública de $\$ 43,000$ por cada $\$ 10,000$ invertidos en intervenciones breves. Aunque son necesarias valoraciones económicas similares en otros países, todo parece indicar que, además de las mejoras en el estatus de la bebida y de las ventajas para la salud y el bienestar de sus destinatarios, las intervenciones breves pueden suponer un importante ahorro para los sistemas de asistencia sanitaria.

Es obvio que resulta necesario realizar más investigaciones sobre la eficacia de las intervenciones breves, incluyendo los mejores métodos para el cribado y la identificación, el modelo óptimo y la longitud de la intervención, y las consecuencias de ser llevadas a cabo por enfermeras y asesores especializados en el tema del alcohol ${ }^{8}$. Así, los métodos de identificación e intervención pueden también precisar ser adaptados a los requisitos de los sistemas de asistencia sanitaria de cada uno de los países y a la eficacia de esos métodos establecidos en las situaciones "reales" de la práctica habitual en los países interesados. Con todo, el balance abrumador de la evidencia a favor de las intervenciones breves justifica en la actualidad la realización de un esfuerzo conjunto para la difusión general y la puesta en práctica de dichas intervenciones breves en la asistencia sanitaria primaria.

\section{El programa de investigación WHO}


Este giro en el objetivo de la investigación hacia las intervenciones breves queda bien ilustrado a través de una serie de estudios reunidos por la Organización Mundial de la Salud bajo el título Proyecto Internacional de Cooperación para la Identificación y el Control de los Problemas relacionados con el Alcohol en la Asistencia Primaria Sanitaria ${ }^{21}$, un programa de investigación que lleva casi veinte años en marcha. En la Fase / de ese proyecto, efectuado en seis países (Australia, Bulgaria, Kenia, Méjico, Noruega y Estados Unidos), se desarrolló un instrumento válido y fiable de cribado para detectar a los bebedores de riesgo o a los que el alcohol resultaba nocivo en el marco de la asistencia sanitaria primaria (el test AUDIT de identificación de trastornos en el consumo de alcohol) ${ }^{22,23}$. El AUDIT se utiliza actualmente en todo el mundo. La Fase // fue un ensayo clínico de cruce de culturas y de múltiples centros en el que participaron diez países (Australia, Bulgaria, Costa Rica, Kenia, Méjico, Noruega, la entonces llamada Unión Soviética, Reino Unido, Estados Unidos y Zimbabwe) ${ }^{15,24}$. En ella se llegó a la conclusión de que, entre los hombres, cinco minutos de "simple asesoramiento" de manos de un consejero sanitario especialmente preparado, basado en un asesoramiento de veinte minutos, tenía como resultado un descenso medio del $25 \%$ en el consumo de alcohol; mientras que en el grupo de control sólo uno de cada cinco pacientes masculinos que habían experimentado intervenciones breves respondía favorablemente. En ese estudio, no se detectaron ventajas adicionales cuando el asesoramiento era más largo.

En la fase III, Ilevada a cabo en catorce países del mundo (incluyendo los europeos siguientes: Bélgica, Bulgaria, Dinamarca, Francia, Hungria, Italia, Noruega, Polonia, Portugal, Federación Rusa y Reino Unido), se estudiaron las prácticas en uso, las percepciones y las actitudes ante las intervenciones breves de los médicos de familia y de los informadores clave seleccionados, lo que condujo al listado de las principales barreras e incentivos para comprometerse en dicha tarea $^{25-27}$. En una fase posterior de la investigación, se evaluaron mediante una serie de tests controlados los métodos para impulsar la comprensión y la utilización del conjunto de las intervenciones breves por parte de los médicos de familia, así como los niveles variables de formación y apoyo. Los resultados de las cuatro ramas de este estudio que ya han sido publicados (Australia ${ }^{28}$, Dinamarca ${ }^{29}$, Nueva Zelanda ${ }^{30}$ y Reino Unido ${ }^{31,32}$ ) se muestran de acuerdo en que el marketing vía teléfono es el medio más rentable de difundir las intervenciones breves entre los médicos de familia, y en que la formación unida a un apoyo telefónico continuado aumenta el porcentaje de su puesta en práctica. Estos estudios, sin embargo, se realizaron en unas condiciones de estrecho control y muchos de los resultados obtenidos no se prolongaron más allá de la duración de las pruebas. Por ese motivo, queda aún por demostrar el mejor medio de impulsar el empleo generalizado y duradero de las intervenciones breves en el campo del alcohol en la práctica habitual.

La fase posterior y posiblemente final de este programa de investigación (Fase IV) está actualmente en marcha y, en Europa, incluye centros de investigación en Flandes, Bulgaria, Cataluña, Dinamarca, Inglaterra, Francia, Finlandia, Hungría, Italia, Letonia, Portugal, Federación Rusa, Eslovenia y Suiza. El objetivo de esta fase es simplemente desarrollar y aplicar estrategias a lo largo y a lo ancho de cada uno de los países que permitan una aplicación generalizada, habitual y duradera de la identificación temprana y de las intervenciones breves en los sistemas sanitarios primarios de los países que participan en el proyecto. La Fase IV intentará también determinar hasta qué punto las estrategias en todo el país lograrán sus objetivos de generalizar esa puesta en práctica, y si eso constituirá una importante contribución para la salud pública y el bienestar general. En contraste con las fases anteriores del proyecto de cooperación WHO, la Fase IV se definiría como una investigación en marcha en la que el objetivo central es establecer una diferencia significativa con las condiciones del "mundo real" bajo las que las intervenciones breves se hallan difundidas y establecer un programa de acción que conduzca a su aplicación general en todo el país. Mientras los estudios individuales de cada país pueden diferir en forma y contenido, todos ellos se adhieren a cuatro componentes básicos: 1) Empleo habitual de los materiales y servicios de las intervenciones breves para ajustarse a las necesidades del sistema de asistencia sanitaria primaria del país en cuestión; 2) "Nueva formulación" de las interpretaciones de los problemas derivados del alcohol, por parte de los profesionales de la asistencia sanitaria y del público en general, a fin de poder aplicar el término de "bebedor de riesgo" a un amplio sector de la población; 3) Desarrollo de una alianza estratégica de organizaciones e individuos con intereses comunes dentro de cada país a fin de favorecer y difundir su aplicación; 4) Proyecto de Demostración con la aspiración de mostrar que la puesta en práctica generalizada puede llevarse a cabo dentro de un área geográfica definida, además de aportar beneficios tangibles a la salud pública. Además de una evaluación cuidadosa de todos los aspectos del estudio, la Fase IV prestará especial atención a una evaluación económica para demostrar fehacientemente que realizar intervenciones breves resulta un método muy útil dentro de los costosos recursos de la asistencia sanitaria.

\section{Programas de cribado sanitario.}

En los países nórdicos se ha desarrollado una tradición diferente de investigación respecto a las intervenciones breves. Se refiere a la eficacia de las intervenciones breves como parte de los programas 
de cribado entre la población. El estudio pionero fue realizado en la década de 1970 y en los primeros años de la década de 1980 por Kristenson y colaboradores entre los residentes varones de Malmö con edades que oscilaban entre 45 y 50 años $^{33,34}$. Comparados con el grupo de control, los pacientes que acudían a tres consultas mensuales con un médico y recibían un feedback mensual sobre unas cifras inicialmente elevadas de gamma-GT más el asesoramiento de una enfermera, mostraron unos niveles de gamma-GT más bajos, además de pasar menos días con síndrome de abstinencia y en el hospital. Con anterioridad a los resultados de la investigación de Fleming y colaboradores $^{19}$, éste ha sido el único estudio que ha demostrado los efectos a largo plazo de la intervención breve, con beneficios detectables hasta cinco años después de la intervención.

También en Suecia, Romelsjö y colaboradores seleccionaron a una serie de individuos para realizar una investigación sanitaria con muestras aleatorias de la población adulta del área de Estocolmo. Ofrecieron a los que bebían en exceso el asesoramiento simple y breve de un médico de familia o un promedio de tres visitas al médico de familia para un feedback de gamma-GT, además de apoyo. En ese estudio, el mejor resultado de la intervención más generalizada no resultó estadísticamente significativo, debido quizá a la naturaleza del grupo que se comparaba (i.e. simple asesoramiento) o al reducido número del muestreo. En un estudio sanitario de más de 21.000 hombres y mujeres en Tromso, Noruega, Nilssen ${ }^{35}$ eligió de forma aleatoria a "bebedores en un primer estadio de riesgo" para un grupo de control y dos grupos (mayor y menor) de intervenciones breves. En los dos grupos de intervención, los pacientes mostraron una reducción mayor de gamma-GT y del consumo declarado por ellos que el grupo de control, pero no parecieron existir diferencias significativas entre las dos formas de intervención. Finalmente, como parte de una encuesta sanitaria general entre hombres finlandeses de edad mediana, Suokas ${ }^{36}$ distribuyó de forma aleatoria los individuos que bebían en exceso entre un grupo que había recibido un asesoramiento breve de manos de un médico y un grupo de intervención que había acudido a tres consultas complementarias con una enfermera a lo largo de un año y que había recibido un feedback de gamma-GT y un folleto de autoayuda. Llegó a la conclusión de que, dada la existencia de daños orgánicos relacionados con el consumo de alcohol, un asesoramiento breve de manos de un médico podía influir favorablemente en los resultados, si bien una intervención más intensiva tenía muy poco que añadir a dicho efecto.

\section{Atención en hospitales generales}

Otro marco evidente en el que desplegar las intervenciones breves es dentro de los hospitales, ya que, entre los pacientes ingresados, existe un elevado número de individuos que beben en exceso ${ }^{37}$ y allí existe una oportunidad mayor de ayudar con intervenciones breves a los pacientes que se sienten preocupados por ello. Un primer estudio de Chick y colaboradores ${ }^{38}$, realizado en Edimburgo, es citado con frecuencia como una prueba ostensible de la eficacia del asesoramiento por parte de una enfermera en el interior del hospital para abstenerse o reducir el consumo de alcohol. Por desgracia, este estudio no prueba dicha evidencia, ya que no existía la menor diferencia entre los niveles de consumo del grupo que había recibido asesoramiento y los del grupo de control, y la única ventaja de la intervención era como medida complementaria ad hoc. En otra investigación llevada a cabo en Nueva Zelanda ${ }^{39}$, un grupo de individuos que bebían en exceso y que habían consultado con un asesor en el tema del alcohol mostraron un consumo significativamente menor durante un año de seguimiento que los grupos de control. No obstante, se trataba de un estudio sobre el proceso de consulta más que de la intervención breve per se.

Posteriormente, un estudio realizado en Sydney, Australia ${ }^{40}$, afirmó que un asesoramiento breve dentro del hospital, de una duración de 30-40 minutos, resultaba eficaz para reducir el consumo de alcohol durante seis meses, al menos entre los pacientes varones en los que se centraba la investigación. Otro resultado de ese estudio fue que los pacientes "predispuestos al cambio", en términos de las fases de modelo de cambio de Prochaska y Di Clemente ${ }^{41}$, se beneficiaban más de un enfoque motivacional al asesoramiento que de un enfoque basado en las habilidades, en tanto que aquellos "predispuestos al cambio" respondían igualmente a los dos tipos de intervención.

Deberíamos añadir que un estudio recientemente finalizado en el Hospital del Kings College, Londres, no logró descubrir el menor efecto de las intervenciones breves entre los pacientes ingresados ${ }^{42}$. Ello podría ser debido a que la duración de la intervención proporcionada en ese estudio (10 minutos) era demasiado breve para tener consecuencias. De ser así, todavía queda por explicar por qué razón el asesoramiento breve parece resultar eficaz para que los pacientes abandonen la bebida en el marco de la asistencia primaria pero no en el marco hospitalario general. Es evidente la necesidad de profundizar en dicha investigación con el fin de identificar las condiciones bajo las que las intervenciones breves podrían resultar eficaces entre los pacientes ingresados en centros hospitalarios.

\section{Servicios de urgencia}

Otro marco médico potencialmente propicio para suministrar intervenciones breves son los servicios de urgencias (Accident and Emergency Services -A\&E- o cualesquiera que sean los nombres que reciban en los 
distintos países); es bien sabido que un porcentaje muy elevado de los que acuden a dichos servicios presentan una intoxicación o, al ser cuestionados, declaran tener algún problema relacionado con el alcohol $^{43-45}$.

Aunque se ha hablado mucho sobre el tema y se han publicado algunos trabajos empíricos sobre las posibilidades del cribado y de la intervención entre los que consumen alcohol de forma abusiva en el marco de los $A \& E^{44,46-49}$, existe un único estudio de un RCT de intervenciones breves. En Finlandia, Antti-Poika ${ }^{50}$ distribuyó de forma aleatoria 120 varones que bebían en exceso y que habían acudido a un servicio de urgencias a causa de sus lesiones entre un grupo de intervención o de control (que recibieron atención ordinaria). El grupo de intervención recibió hasta tres sesiones de asesoramiento de un auxiliar de enfermería especialmente preparado. Los resultados fueron alentadores: a los seis meses del tratamiento, el $45 \%$ de los pacientes del grupo de intervención habían moderado o abandonado el consumo de alcohol, frente al $20 \%$ del grupo de control, una diferencia estadísticamente significativa reforzada por los cambios en las cifras de gamma-GT. Este estudio parece sugerir que las intervenciones breves pueden ser eficaces en el marco de los A\&E. En el Reino Unido y probablemente en otros países se están realizando más investigaciones sobre dicha posibilidad.

\section{Otros marcos médicos}

Hay algunos otros marcos médicos en los que se han estudiado las intervenciones breves. Por ejemplo, a partir de los datos recogidos en una clínica somática diurna en Lund, Suecia, Persson y Magnussen ${ }^{51}$ distribuyeron de forma aleatoria a individuos que bebían en exceso entre grupos de intervenciones breves y grupos de control. Llegaron a la conclusión de que una intervención relativamente simple era efectiva en ese marco y podría ser llevada a cabo a un coste moderado. Un estudio sobre las intervenciones breves realizado en una clínica de hipertensión en Inglaterra llegó a una conclusión muy parecida ${ }^{52}$. Aquellos pacientes a los que un médico proporcionó un asesoramiento breve para reducir drásticamente la bebida mostraron una reducción significativamente mayor que el grupo de control en el consumo de alcohol, gamma-GT y presión arterial diastólica.

Por último, Chang y colaboradores ${ }^{53}$ evaluaron en Estados Unidos el impacto de la intervención breve durante la gestación entre mujeres identificadas como consumidoras abusivas de alcohol en las revisiones ginecológicas. En esa ocasión, sin embargo, no se detectó una superioridad significativa de la intervención breve sobre el grupo de control, debido quizá a los efectos del asesoramiento general de dos horas de duración que recibieron los individuos del grupo de control.

\section{La intervención breve para reducir el consumo de riesgo del alcohol entre los jóvenes: el papel de los profesionales de la asistencia sanitaria}

La segunda parte de este artículo centrará su atención en el consumo de riesgo del alcohol entre los jóvenes y explorará el potencial de las intervenciones breves en el marco de la asistencia sanitaria, a fin de reducir los daños relacionados con el alcohol, concretamente entre este sector de población. En primer lugar, no obstante, se intentará caracterizar la naturaleza del consumo de riesgo del alcohol entre los jóvenes y contemplar las implicaciones de una forma de consumo tan diferente en lo que se refiere a las consecuencias sanitarias y sociales, tanto para los jóvenes como para las demás personas a quienes afecta su forma de beber.

\section{El consumo de alcohol entre los jóvenes}

A pesar de que beber en exceso es una causa importante de mortalidad, morbididad y problemas sociales en todo el mundo, su impacto no está distribuido equitativamente entre las poblaciones ${ }^{54}$. Los problemas relacionados con el alcohol son experimentados con mayor frecuencia y severidad por los individuos con un estatus socioeconómico más bajo ${ }^{5-56} \mathrm{y}$ por los jóvenes ${ }^{57}$. Esta experiencia diferencial de los problemas relacionados con el alcohol no puede justificarse únicamente en base a las diferencias en los niveles medios de consumo ${ }^{54}$ y se ha planteado que los modelos de beber alcohol pueden también ejercer cierta influencia. El consumo de diferentes bebidas alcohólicas, vinculado a determinadas formas de consumo, puede acarrear problemas diferenciales sanitarios y sociales, especialmente entre los jóvenes ${ }^{59}$.

Los niveles de consumo entre los jóvenes son más altos que entre el resto de la población. Tomando a Inglaterra y a Gales como ejemplo, los porcentajes de jóvenes de ambos sexos (entre 16 y 24 años) que bebían por encima de los niveles recomendables para la salud eran del 35\% (varones) y del 21\% (mujeres), comparados con el $27 \%$ y el $13 \%$, respectivamente, del total de la población adulta ${ }^{60}$. Recientemente se ha conocido el dato de que el $47 \%$ y el $36 \%$ de los chicos y de las chicas ingleses entre 11 y 15 años beben alcohol semanalmente ${ }^{61}$. Puesto que los jóvenes no suelen beber con la misma frecuencia que los adultos, este aumento en el consumo es a menudo el resultado de beber con mayor intensidad y riesgo en una única ocasión ${ }^{57}$, o de beber hasta emborracharse ${ }^{62}$, un modelo de consumo de alcohol fuertemente asociado con la intoxicación y la embriaguez. Un informe WHO muy reciente sobre la actitud ante la salud de jóvenes de 27 países hablaba de una tendencia cada mayor a beber hasta emborracharse, aunque los porcentajes de los estados de embriaguez reconocidos por los propios bebedores solían aumentar con la edad y eran 
más frecuentes entre los chicos que entre las chicas ${ }^{61}$. Sin embargo, también aseguraba que en Inglaterra, Escocia, Groenlandia y Finlandia, las chicas de quince años relataban episodios de embriaguez con más frecuencia que los chicos de su misma edad ${ }^{61}$.

El hecho de beber hasta emborracharse va asociado entre los jóvenes al inicio temprano de relaciones sexuales ${ }^{63}$, así como de los riesgos que ello comporta: contraer enfermedades de transmisión sexual y embarazos no deseados ${ }^{57}$. Beber hasta embriagarse juega también un importante papel en los incidentes de asalto y violación entre los jóvenes que habitan en ambientes estudiantiles ${ }^{64}$. Ha sido, asimismo, vinculado a una incidencia mayor de accidentes de tráfico y de actividades delictivas ${ }^{57}$, incluyendo el conducir en estado de embriaguez o ir de pasajero con un conductor ebrio ${ }^{63}$. Un rasgo significativo de los problemas relacionados con el alcohol que están ligados a la salud sexual y a los traumas es que a menudo repercuten en otros individuos que no son el propio bebedor. Weschler y colaboradores ${ }^{66}$ afirmaron que los estudiantes que elegían no emborracharse pero vivían en algún campus con un elevado número de jóvenes que sí lo hacían padecían más asaltos, insinuaciones sexuales no deseadas y perturbaciones en sus estudios como resultado de ello. Además, el hecho de beber hasta embriagarse está estrechamente asociado entre los jóvenes al consumo de tabaco ${ }^{61,67}$, dificultades de comunicación con las familias ${ }^{61}$, problemas académicos tempranos ${ }^{61}$ y trastornos mentales ${ }^{68}$. Es bien sabido que los comportamientos problemáticos y los riesgos para la salud se dan con frecuencia entre los jóvenes ${ }^{69}$ y que existen asociaciones muy fuertes entre el consumo de sustancias, incluyendo el alcohol, y otros comportamientos de riesgo para la salud ${ }^{70}$.

Posibilidades de intervención breve en el campo del alcohol entre los jóvenes

Cuando beber hasta embriagarse ocasiona algún trauma, los jóvenes suelen presentarse en los servicios de urgencia ${ }^{71}$. Una serie de estudios recientemente realizados en el Reino Unido han puesto de manifiesto la viabilidad ${ }^{72-74}$ y el impacto de la intervención breve en el campo del alcohol en el marco de los $A \& E^{75}$. Aunque ello supone una clara oportunidad de intervenir, especialmente entre los varones jóvenes que consumen alcohol de forma abusiva, estos estudios sugieren que pueden darse importantes problemas logísticos y culturales asociados con la intervención en el campo del alcohol en los $A \& E^{72-73}$. Dichos problemas necesitan ser solucionados antes de que el potencial de las intervenciones breves en el marco de los A\&E pueda hacerse realidad.

Un marco alternativo de la asistencia sanitaria para la detección de los problemas relacionados con el alcohol entre los jóvenes es la asistencia sanitaria primaria. Los jóvenes acuden habitualmente a la asisten- cia primaria para inscribirse como nuevos pacientes, promoción de la salud (incluyendo inmunización), servicios de salud sexual (incluyendo anticonceptivos de emergencia), trastornos mentales y tratamiento de traumas menores ${ }^{76}$. Esos contextos proporcionan un campo muy amplio para el examen médico preventivo y la intervención breve en los problemas relacionados con el alcohol.

Que existe una necesidad demostrable de cribado e intervención breve entre los jóvenes dentro de la asistencia primaria queda evidenciado por el reciente estudio efectuado en el nordeste de Inglaterra ${ }^{77}$. Según esta investigación, el consumo de alcohol de 12,814 pacientes fue oportunamente chequeado por médicos de familia empleando el cuestionario AUDIT ${ }^{31-}$ 32. Una quinta parte de los pacientes bebían de forma abusiva y el $22 \%$ de ellos tenían entre 16 y 24 años. A pesar de que muchos de esos jóvenes bebedores de riesgo afirmaron sentirse culpables o arrepentirse de haber consumido alcohol (21\%), no ser capaces de recordar lo ocurrido la noche anterior a causa de la bebida (26\%) y haberse causado daño físico a sí mismos o a los demás por el exceso de alcohol (10\%), sólo el $5 \%$ de ellos reconocieron haber recibido asesoramiento de algún profesional de la sanidad o de alguna persona "preocupada" por su actitud ante la bebida $^{77}$.

Tal como hemos detallado antes, la eficacia y la rentabilidad de la intervención breve en la asistencia primaria resultan evidentes para reducir el consumo abusivo de alcohol. Sin embargo, el impacto de la intervención breve en el campo del alcohol no parece tan claro entre los jóvenes. Se ha observado que a menudo han faltado jóvenes en las pruebas destinadas a evaluar la eficacia de la intervención breve en los marcos sanitarios ${ }^{78}$. Además, un estudio reciente ha encontrado que sólo la mitad de los identificados como bebedores abusivos habían recibido intervención breve de un médico de familia y que los jóvenes estaban entre los bebedores de riesgo con menos posibilidades de recibir dicha intervención ${ }^{32,79}$. Por ese motivo, es preciso realizar más estudios para determinar la eficacia de la intervención breve en el campo del alcohol entre los jóvenes que beben hasta emborracharse, especialmente en la asistencia sanitaria primaria.

Por otra parte, las intervenciones breves en el campo del alcohol realizadas por profesionales de la sanidad han tendido a centrarse en los individuos que consumen alcohol de forma abusiva y con frecuencia (que son normalmente adultos), asesorándoles sobre el mejor modo de reducir los niveles medios de consumo, normalmente a base de incrementar el número de días de abstinencia por semana. Los jóvenes que beben hasta estar ebrios, sin embargo, parecen necesitar un consejo muy diferente del que suele darse en las sesiones estándar de intervención breve. Quizá 
éste debería centrarse en reducir el número y la mezcla de bebidas consumidas en una única salida o en minimizar los riesgos de la intoxicación. Es posible que una intervención efectiva para reducir los efectos negativos del consumo de alcohol entre los jóvenes necesite dirigirse a otras áreas de riesgo para la salud (ej, riesgo sexual) a fin de minimizar los daños. Adoptar semejante enfoque de reducción de daños del consumo de alcohol hasta emborracharse resultaría coherente con las estrategias empleadas con éxito en el campo de las drogas ilegales durante muchos años ${ }^{80}$, y que han sido últimamente defendidas en relación con el alcohol ${ }^{81-82}$.

Para finalizar, una intervención efectiva entre los jóvenes bebedores precisará tener en cuenta las funciones positivas del alcohol ${ }^{83}$, incluyendo motivos como el de poder hacer frente a la presión social y emocional que los jóvenes asocian a la conducta de beber $^{84}$ y de acudir a los lugares públicos donde se consume alcohol ${ }^{85}$. Esos factores parecen jugar un papel importante a la hora de decidir el balance motivacional para el cambio en los jóvenes bebedores de riesgo. Lo cierto es que se ha planteado que el asesoramiento adaptado a diferentes clases de bebedores y estadios de motivación para el cambio puede aumentar el impacto de la intervención en el alcohol ${ }^{86}$, y la evidencia del cese en el campo del tabaco sugiere que se trata de un enfoque muy prometedor para investigaciones futuras ${ }^{87}$. Por otra parte, algunos estudios realizados a pequeña escala en el marco escolar y comunitario se han aproximado al tema mediante el empleo de intervenciones especialmente adaptadas ${ }^{88}$ y de entrevistas motivacionales con resultados positi$\operatorname{vos}^{89}$, aunque limitados, entre los jóvenes bebedores de riesgo. Dados los problemas motivacionales especiales inherentes a los intentos de los adultos por cambiar el comportamiento de los jóvenes, las entrevistas motivacionales, en las que las motivaciones más que imponerse de algún modo a los entrevistados surgen de ellos, parecen ofrecer más oportunidades para designar las intervenciones breves eficaces entre esa población, aunque debe reconocerse que esa forma de intervención breve será inevitablemente más larga que la ofrecida habitualmente a los adultos que consumen demasiado alcohol (de 5-10 minutos) y es muy probable que exija la presencia de asesores especialmente hábiles y bien preparados para su puesta en práctica.

Hasta la fecha, la mayoría de las investigaciones sobre el uso incorrecto de la prevención del alcohol entre los jóvenes han tenido lugar en el marco educacional. Sin embargo, un reciente análisis sistemático de la literatura publicada ha acusado a esa parte del estudio de tener importantes defectos metodológi$\cos ^{90}$. A pesar de ello, el reconocimiento de que el consumo de riesgo del alcohol entre los jóvenes, y especialmente el hecho de beber hasta emborrachar- se, es un importante problema para la salud pública, que repercute negativamente no sólo en el bebedor sino también en otras personas, obliga a los estudiosos de la asistencia sanitaria a efectuar evaluaciones científicas cuidadosamente diseñadas de las intervenciones breves, con el fin de reducir los daños relacionados con el alcohol entre ese sector de la población.

\section{Conclusiones}

Desde este análisis de la literatura sobre la eficacia de las intervenciones breves en el marco de la asistencia sanitaria para reducir el consumo abusivo de alcohol, las siguientes conclusiones parecen justificadas:

\section{Intervenciones breves en general}

1. En términos generales, las intervenciones breves proporcionadas en el marco de la asistencia sanitaria resultan eficaces para reducir el consumo de alcohol entre los que beben en exceso (bebedores de riesgo y bebedores a los que el alcohol resulta nocivo) y resultarían claramente beneficiosas para la salud pública si se pusieran en práctica de forma seria y generalizada dentro de ese marco.

2. Desgraciadamente, los profesionales de la asistencia sanitaria han sido lentos a la hora de incorporar en su práctica habitual las intervenciones breves en el campo del alcohol y es necesario realizar más esfuerzos para animarlos a acometer dicha tarea.

3. La eficacia de las intervenciones breves entre pacientes hombres y mujeres es claramente mayor en el marco de la asistencia primaria. Ello incluye en la actualidad efectos de intervención que duran hasta cuatro años, además del descubrimiento de importantes beneficios económicos para el sistema de asistencia sanitaria.

4. Una secuencia experimental de investigación sobre las intervenciones breves en el campo del alcohol dentro de la asistencia sanitaria primaria queda ilustrada por el programa de investigación WHO titulado: Proyecto Internacional de Cooperación para la Identificación y el Control de los Problemas relacionados con el Alcohol dentro de la Asistencia Sanitaria Primaria. La última fase de este programa (Fase IV) consta de una serie de proyectos activos de investigación cuyo objetivo es la puesta en práctica generalizada, habitual y duradera de las intervenciones breves en el campo del alcohol dentro de los sistemas de asistencia sanitaria primaria de los distintos países que intervienen en el programa. 
5. Una línea tradicional de investigación en los países nórdicos se ha ocupado de la eficacia de las intervenciones breves como parte de los programas de cribado entre la población. Ello también ha servido para probar la efectividad de las intervenciones breves en el campo del alcohol, incluyendo los efectos a largo plazo.

6. La eficacia de las intervenciones breves entre los pacientes ingresados en un hospital no es tan clara como se pensaba. Es preciso realizar más investigaciones para identificar las formas más efectivas de cribado e intervención breve necesarias dentro de este marco.

7. Los servicios de urgencia parecen lugares muy prometedores para el cribado y las intervenciones breves entre los consumidores abusivos de alcohol, pero los estudios realizados sobre dicha posibilidad están aún en sus inicios.

8. Resultarían de enorme utilidad nuevos estudios sobre la eficacia y el impacto de las intervenciones breves en otros marcos de la asistencia sanitaria.

\section{Intervenciones breves entre los jóvenes}

9. Los jóvenes experimentan cada vez con mayor frecuencia y severidad los problemas relacionados con el alcohol. Ello se debe no sólo a niveles más elevados de consumo que en grupos de otras edades, sino también a determinadas modas de consumo entre los jóvenes, especialmente la de "beber hasta emborracharse".

10. El hecho de que los jóvenes beban de ese modo se asocia a una serie de problemas, incluyendo el inicio temprano de relaciones sexuales, enfermedades de transmisión sexual, embarazos no deseados, asaltos y violaciones, accidentes de tráfico y otras actividades delictivas, elevada prevalencia del consumo de tabaco, problemas de comunicación con sus familias, dificultades académicas tempranas y trastornos mentales.

11. A pesar de que los servicios de urgencia ofrecen una buena oportunidad para intervenir, especialmente entre los jóvenes varones que consumen alcohol de forma abusiva y que puedan haber sufrido algún trauma relacionado con la bebida, es probable que existan importantes dificultades logísticas y culturales que sea preciso solucionar antes de que el potencial de las intervenciones breves en el marco de los A\&E pueda hacerse realidad.

12. Un marco alternativo de la asistencia sanitaria para la detección y el control de los problemas relacionados con el alcohol entre jóvenes de ambos sexos es la asistencia primaria. Las oportunidades específicas para el cribado y la intervención breve engloban el registro de los nuevos pacientes, la promoción de la salud (incluyendo inmunización), los servicios de salud sexual (incluyendo anticonceptivos de emergencia), los trastornos mentales y el tratamiento de traumas menores. No obstante, es urgente profundizar en el estudio de esas posibilidades.

13. Es probable que las intervenciones breves eficaces entre los jóvenes necesiten prestar especial atención a: I) El estilo específico de consumo abusivo de alcohol mostrado por los jóvenes (beber en pocas ocasiones hasta emborracharse, en lugar de beber en exceso de forma regular) y II)Los problemas motivacionales de carácter personal tan relevantes entre los jóvenes, incluyendo las funciones sociales y emocionales de la bebida. La forma general de intervención conocida como "entrevistas motivacionales", aunque parece precisar más tiempo que las intervenciones breves entre los adultos, además de asesores más capacitados, ofrece mejores perspectivas a la hora de planificar las intervenciones breves entre ese sector de población.

14. Unas evaluaciones científicas cuidadosamente diseñadas de las intervenciones breves para reducir los daños relacionados con el alcohol entre los jóvenes deberían constituir una prioridad en los futuros programas de investigación nacionales e internacionales.

\section{BIBLIOGRAFÍA}

1. Bien, T.H., Miller, W.R., \& Tonigan, J. (1993). Brief interventions for alcohol problems: A review. Addiction, 88, 315-336.

2. Fremantle, N., Gill, P., Godfrey, C., Long, A., Richards, C., Sheldon, T., Song, F., \& Webb, J. (1993). Brief interventions and alcohol use. Effective Health Care Bulletin \#7. Nuffield Institute for Health: Leeds, UK.

3. Heather, N. (1995). Brief intervention strategies. In R.K. Hester \& W.R. Miller (Eds.), Handbook of Alcoholism Treatment Approaches: Effective Alternatives. (2nd edition). Needham Heights MS: Allyn \& Bacon.

4. Kahan, M., Wilson, L. \& Becker, L. (1995). Effectiveness of physician-based interventions with problem drinkers: a review. Canadian Medical Association Journal, 152, 851-859.

5. Wilk, A.I., Jensen, N.M. \& Havighurst, T.C. (1997). Metaanalysis of randomised control trials addressing brief interventions in heavy alcohol drinkers. Archives of Internal Medicine, 12, 274-283.

6. Wallace, P., Cutler, S., \& Haines, A. (1988). Randomised controlled trial of general practitioner intervention in patients with excessive alcohol consumption. British Medical Journal, 297, 663-668. 
7. Heather, N. (1996). The public health and brief interventions for excessive alcohol consumption: the British experience. Addictive Behaviors, 21, 857-868.

8. Heather, N. (in press). Brief interventions. In N. Heather, T. Peters \& T. Stockwell (Eds.), International Handbook of Alcohol Dependence and Problems. Chichester, UK: John Wiley.

9. Romelsjö, A., Andersson, L. Barrner, H. et al. (1989). A randomised study of secondary prevention of early stage problem drinkers in primary health care. British Journal of Addiction, 84, 1319-1327.

10. Anderson, P. \& Scott, E. (1992). The effect of general practitioners' advice to heavy drinking men. British Journal of Addiction, 87, 891-900.

11. Richmond, R., Heather, N., Wodak, A., Kehoe, L. \& Webster, I. (1995). Controlled evaluation of a general practice-based brief intervention for excessive drinking. Addiction, 90, 119-132.

12. Fleming, M.F., Barry, K.L., Manwell, L.B., Johnson, K. \& London, R. (1997). Brief physician advice for problem alcohol drinkers: A randomised controlled trial in community-based primary care practices. Journal of the American Medical Association, 277, 1039-1045.

13. Senft, R.A., Polen, M.R., Freeborn, D.K. \& Hollis, J.F. (1997). Brief intervention in a primary care setting for hazardous drinkers. American Journal of Preventive Medicine, 13, 464-70.

14. Córdoba, R., Delgado, M.T., Pico, V. et al. (1998). Effectiveness of brief intervention on non-dependent alcohol drinkers (EBIAL): a Spanish multi-centre study. Family Practice, 15, 562-567.

15. Babor, T.F. \& Grant, M. (Eds.) (1992). Project on Identification and Management of Alcohol-related Problems. Report on Phase II: A Randomised Clinical Trial of Brief Interventions in Primary Health Care. Geneva: World Health Organization.

16. Israel, Y., Hollander, O., Sanchez-Craig, M. et al. (1996). Screening for problem drinking and counseling by the primary care physician-nurse team. Alcoholism: Clinical \& Experimental Research, 20, 1443-1450.

17. Ockene, J.K., Adams, A., Hurley, T.G., Wheeler, E.V., \& Herbert, J.R. (1999). Brief physician- and nurse practitioner-delivered counseling for high-risk drinkers: does it work? Archives of Internal Medicine, 159, 21982205.

18. Scott, E. \& Anderson, P. (1991). Randomised controlled trial of general practitioner intervention in women with excessive alcohol consumption. Drug \& Alcohol Review, 10, 313-321.

19. Fleming, M.F., Mundt, M.P., French, M.T., Manwell, L.B., Stauffacher, E.A. \& Barry, K.L. (2000). Brief physician advice for problem drinkers: long-term efficacy and benefit-cost analysis. Unpublished ms., Department of Family Medicine, University of Wisconsin-Madison, WI 53715, USA.

20. Fleming, M.F., Mundt, M.P., French, M.T., Manwell, L.B., Stauffacher, E.A. \& Barry, K.L. (2000). Benefit-cost analysis of brief physician advice with problem drinkers in primary care settings. Medical Care, 38, 7-18.

21. Monteiro, M.G. \& Gomel, M. (1998). World Health Organization project on brief interventions for alcoholrelated problems in primary health care settings. Journal of Substance Abuse, 3, 5-9.

22. Babor, T.F., de la Fuente, J.R., Saunders, J.B. \& Grant, M (1989). AUDIT - The Alcohol Use Disorders Identification Test: Guidelines for Use in Primary Health Care. Geneva: World Health Organization.

23. Saunders, J.B. \& Aasland, O.G. (1987). WHO Collaborative Project on the Identification and Treatment of Persons with Harmful Alcohol Consumption. Report on Phase I: Development of a Screening Instrument. Geneva: World Health Organization.

24. Babor, T.F., Grant, M. et al. (1994). A randomised clinical trial of brief interventions in primary health care: summary of a WHO project. Addiction, 89, 657-678.

25. Saunders, J. \& Wutzke, S. (Eds.) (1998). WHO Phase III Collaborative Study on Implementing and Supporting Intervention Strategies in Primary Health Care. Report on Strand 1: General Practitioners' Current Practices and Perceptions of Preventive Medicine and Intervention for Hazardous Alcohol Use. Copenhagen, Denmark: WHO Regional Office for Europe, Alcohol, Drugs \& Tobacco Programme.

26. Kaner E., Heather N. \& McAvoy B. et al. (1999). Intervention for excessive alcohol consumption in primary health care: attitudes and practices of English general practitioners. Alcohol \& Alcoholism, 34, 55966.

27. McAvoy, B.R., Donovan, R.J., Jalleh, G. et al. (in press). General practitioners, prevention and alcohol - a powerful cocktail? Facilitators and inhibitors of practising preventive medicine in general and early intervention for alcohol in particular: a twelve nation key informant and general practitioner study. Drugs: Education, Prevention \& Policy.

28. Gomel, M.K., Wutzke, S.E., Hardcastle, D.M., Lapsley, H. \& Reznik, R. (1998). Cost-effectiveness of strategies to market and train primary health care physicians in brief intervention techniques. Social Science \& Medicine, 47, 203-211.

29. Hansen, L.J., de Fine Olivarius, N., Beich, A. \& Barfod, S. (1999). Encouraging GPs to undertake screening and a brief intervention in order to reduce problem drinking: a randomised controlled trial. Family Practice, 16, 551557.

30. McCormick, R., Adams, P., Powell, A., Bunbury, D., Paton-Simpson, G. \& McAvoy, B. (1999). Encouraging general practitioners to take up screening and early intervention for problem use of alcohol: a marketing trial. Drug \& Alcohol Review, 18, 171-177.

31. Lock, C.A., Kaner, E.F.S., Heather, N., McAvoy, B.R. \& Gilvarry, E. (1999). A randomised trial of three marketing strategies to disseminate a screening and brief alcohol intervention programme to General 
Practitioners. British Journal of General Practice, 49, 695-698

32. Kaner, E.F.S., Haighton, C.A., McAvoy, B.R., Heather, N. \& Gilvarry, E. (1999). A RCT of three training and support strategies to encourage implementation of screening and brief alcohol intervention by general practitioners. British Journal of General Practice, 49, 699-703.

33. Kristenson, H., Ohlin, H., Hulten-Nosslin, M., Trell, E. \& Hood, B. (1983). Identification and intervention of heavy drinking in middle-aged men: results and followup of 24:60 months of long-term study with randomised controls. Alcoholism: Clinical \& Experimental Research, 20, 203-209.

34. Trell, E., Kristenson, H. \& Fex, G. (1984). Alcohol-related problems in middle-aged men with elevated serum gamma-glutamyltransferase: a preventive medical investigation. Journal of Studies on Alcohol, 45, 302309.

35. Nilssen, O. (1991). The Tromsø study: identification of and a controlled intervention on a population of earlystage risk drinkers. Preventive Medicine, 20, 518-528.

36. Suokas, A. (1992). Brief Intervention of Heavy Drinking in Primary Health Care: Hämeenlinna Study. Academic Dissertation, Research \& Treatment Unit for Alcohol Diseases, University of Helsinki: University Printing House.

37. Mclntosh, I.D. (1982). Alcohol-related disabilities in general hospital patients: a critical review of the evidence. International Journal of the Addictions, 17, 609-639.

38. Chick, J., Lloyd, G., \& Crombie, E. (1985). Counselling problem drinkers in medical wards: a controlled study. British Medical Journal, 83, 159-170.

39. Elvy, G.A., Wells, J.E., \& Baird, K.A. (1988). Attempted referral as intervention for problem drinking in the general hospital. British Journal of Addiction, 83, 83-89.

40. Heather, N., Rollnick, S., Bell, A., \& Richmond, R. (1996). Effects of brief counselling among male heavy drinkers identified on general hospital wards. Drug \& Alcohol Review, 15, 29-38.

41. Prochaska, J.O. \& DiClemente, C.C. (1992). Stages of change in the modification of problem behaviors. In M. Hersen, R.M., Eisler \& P.M. Miller (Eds.), Progress in Behavior Modification. Newbury Park, CA: Sage.

42. Marshall, J., Canning, U.P., Kennell-Webb, S.A., Gunstone, C.M., Wessely, S.C. \& Peters, T.J. (2000). The effects of brief intervention amongst "at risk" drinkers on general medical wards: a randomised controlled trial. Paper presented at Annual Symposium of Society for the Study of Addiction to Alcohol and Other Drugs, Leeds, UK, November $3^{\text {rd }}$.

43. Holt, S., Stewart, I.C., Dixon, J.M.J., Elton, R.A., Taylor, T.V., \& Little, K. (1980). Alcohol and the emergency service patient. British Medical Journal, 281, 638-640.

44. Lockhart, S.P., Carter, Y.H., Straffen, A.M., Pang, K.K., McLoughlin, J., \& Baron, J.H. (1986). Detecting alcohol consumption as a cause of emergency general medical admissions. Journal of the Royal Society of Medicine, 79, 132-136.

45. Weisner, C. \& Schmidt, L. (1993). Alcohol and drug problems among diverse health and social service populations. American Journal of Public Health, 83, 824-829.

46. Green, M., Setchell, J., Hames, P., Stiff, G., Touquet, R. \& Priest, R. (1993). Management of alcohol abusing patients in accident and emergency departments. Journal of the Royal Society of Medicine, 86, 393-395.

47. Gentilello, L.M., Donovan, D.M., Dunn, C.W. \& Rivara, F.R. (1995). Alcohol interventions in trauma centers: current practice and future directions. Journal of the American Medical Association, 274, 1043-1048.

48. Smith, S.G.T., Touquet, R., Wright, S. \& Das Gupta, N. (1996). Detection of alcohol misusing patients in accident and emergency departments: the Paddington Alcohol Test (PAT). Journal of Accident \& Emergency Medicine, 13, 308-312.

49. Dunn., C.W., Donovan, D.M. \& Gentilello, L.M. (in press). Practical guidelines for performing alcohol interventions in trauma centers. Journal of Trauma: Injury, Infection \& Critical Care.

50. Antti-Poika, I, Karaharju, E, Roine, R. \& Salaspuro, M. (1988) Intervention of heavy drinking: a prospective and controlled study of 438 consecutive injured male patients. Alcohol \& Alcoholism, 23, 115-21.

51. Persson, J. \& Magnusson, P. (1989) Early intervention in patients with excessive consumption of alcohol: a controlled study. Alcohol, 6, 403-408.

52. Mahaswaren, R, Beevers, M. \& Beevers, DG. (1992) Effectiveness of advice to reduce alcohol consumption in hypertensive patients. Hypertension, 19, 79-84.

53. Chang, G, Wilkins-Haug, L, Berman, S. \& Goetz, MA. (1999) Brief intervention for alcohol use in pregnancy: a randomised trial. Addiction, 94, 1499-1508.

54. Marmot, M. (1997). Inequality, deprivation and alcohol use. Addiction, 92, S13-20.

55. Makela, P., Valkonen, T. \& Martelin. T. (1997). Contribution of deaths related to alcohol use of socio-economic variation in mortality: register based follow-up study. British Medical Journal, 315, 211-216.

56. Van Oers. J., Bongers, I., Van der Goor, L. \& Garretsen, H. (1999). Alcohol consumption, alcohol-related problems, problem drinking and socio-economic status. Alcohol \& Alcoholism, 34, 78-88.

57. Murgraff, V., Parrott, A. \& Bennett, P. (1999). Risky singleoccasion drinking amongst young people: definition, correlates, policy and intervention. Alcohol \& Alcoholism, 34, 3-14.

58. Rehm, J. (1996). On the emerging paradigm of drinking patterns and their social and health consequences. Addiction, 91, 1615-1626.

59. Smart, R.G. (1996). Behavioural and social consequences related to the consumption of different beverage types. Journal of Studies on Alcohol, 57, 7784. 
60. Hedges, B. \& di Salvo, P. (1996) Alcohol consumption and smoking. Chapter 8 in Health Survey for England. London: The Stationery Office.

61. Currie, C., Hurrelmann, K., Settertobulte, W., Smith, R. \& Todd, J. (2000). Health and Health Behaviour Among Young People. Copenhagen: WHO Regional Office for Europe.

62. Holmila, M. (1995). Intoxication and hazardous use of alcohol: results from the 1992 Finnish Drinking Habits Study. Addiction, 90, 785-792.

63. Bailey, S.L., Pollock, N.K., Martin, C.S. \& Lynch, K.G. (1999). Risky sexual behaviours among adolescents with alcohol use disorders. Journal of Adolescent Health, 25, 179-81.

64. Koss, MP. \& Gaines, JA. (1993). The prediction of sexual aggression by alcohol use, athletic participation and fraternity affiliation. Journal of Interpersonal Violence, 8, 94-108.

65. Weschler, H. \& Issac, N. (1992). Binge drinking at Massachusetts colleges: prevalence, drinking styles, time trends and associated problems. Journal of the American Medical Association, 267, 2929-2931.

66. Weschler, H, Moeykens, B, Davenport, A, Castillo, S. \& Hansen, J. (1995). The adverse impact of heavy episode drinkers on other college students. Journal of Studies on Alcohol, 56, 628-634.

67. Johnson, P.B., Boles, S.M., Vaughan, R. \& Kleber, H.D. (2000). The co-occurrence of smoking and binge drinking in adolescence. Addictive Behaviours, 25, 779783.

68. Kandel, D., Johnson, J., Bird, H. et al. (1999). Psychiatric comorbidity among adolescents with substance use disorders: findings from the MECA study. Journal of the American Academy of Child \& Adolescent Psychiatry, 38, 693-699.

69. Jessor, R. (1987). Problem Behaviour Theory, psychological development and adolescent problem drinking. British Journal of Addiction, 82, 331-342.

70. DuRant, R.H., Smith, J.A., Kreiter, S.R. \& Krowchick, D.P. (1999). The relationship between early onset of initial substance use and engaging in multiple health risk behaviour. Archives of Pediatrics \& Adolescent Medicine, 153, 286-291.

71. Thom, B., Herring, R. \& Judd A. (1999). Identifying alcohol-related harm in young drinkers: the role of Accident and Emergency Departments. Alcohol \& Alcoholism, 34, 910-915.

72. Peters, J., Brooker, C., McCabe, C. \& Short, N. (1998). Problems encountered with opportunistic screening for alcohol-related problems in patients attending an accident and emergency department. Addiction, 93, 589-594.

73. Brooker, C., Peters. J., McCabe. C. \& Short, N. (1999). The views of nurses to the conduct of a randomised controlled trial of problem drinkers in an accident and emergency department. International Journal of Nursing Studies, 36, 33-39.
74. Smith, A.J., Shepard, J.P. \& Hodgson, R.J. (1998). Brief interventions for patients with alcohol-related trauma. British Journal of Oral \& Maxillofacial Surgery, 36, 408415.

75. Wright, S., Moran, L., Meyrick, M., O'Connor R. \& Touquet, R. (1998). Intervention by an alcohol health worker in an accident and emergency department. Alcohol \& Alcoholism, 33, 651-656.

76. McCormick, A., Fleming, D. \& Charlton, J. (1995). Morbidity Statistics from General Practice: 4th National Study 1991-1992. Series MB5 no.3. London: Office of Population Censuses and Surveys, HMSO.

77. Kaner, E.F.S., Lock, C.A., Heather, N. \& McAvoy, B.R. (2000). Drinking patterns and sociodemographic status in patients screened for excessive drinking by GPs. Unpublished ms, Department of Primary Health Care, University of Newcastle upon Tyne, UK.

78. Edwards, A.G.K. \& Rollnick, S. (1997). Outcome studies of brief alcohol intervention in general practice: the problem of lost subjects. Addiction, 92, 1699-1704.

79. Kaner, E.F.S., Heather, N., Brodie, J., Lock, C.A. \& McAvoy, B. (2000). Sociodemographic status of patients predicts the offer of brief alcohol intervention by GPs. Unpublished ms, Department of Primary Health Care, University of Newcastle upon Tyne, UK.

80. Plant, M. \& Plant, M. (1992). Risk Takers: Alcohol, Drugs, Sex and Youth. London: Tavistock/Routledge.

81. Giesbrecht, N. (1999) Reducing risks associated with drinking among young adults: promoting knowledgebased perspectives and harm reduction strategies. Addiction, 94, 353-355.

82. Mosher, J. (1999). Alcohol policy and the young adult: establishing priorities, building partnerships, overcoming barriers. Addiction, 94, 357-369.

83. Wilsnack, R.W., Vogeltanz, N.D., Wilsnack, S.C. et al. (2000). Gender differences in alcohol consumption and adverse drinking consequences: cross-cultural patterns. Addiction, 95, 251-265.

84. Bradizza, C.M., Reifman, A. \& Barnes, G.M. (1999). Social and coping reasons for drinking: predicting alcohol misuse in adolescents. Journal of Studies on Alcohol, 60, 491-499.

85. Engels, R.C.M.E., Knibbe, R.A. \& Drop, M.J. (1999). Visiting public drinking places: an explorative study into the functions of pub-going for late adolescents. Substance Use \& Misuse, 34, 1261-1280.

86. Rollnick, S., Butler, C. \& Hodgson, R. (1997). Brief alcohol intervention in medical settings: concerns from the consulting room. Addiction Research, 5, 331-342.

87. Dijkstra, A., De Vries, H. \& Roijackers, J. (1999). Targeting smokers with low readiness to change with tailored and non-tailored self-help materials. Preventive Medicine, 28, 203-211.

88. Borsari, B. \& Carey, K.B. (2000) Effects of a brief motivational intervention with college student drinkers. Journal of Consulting and Clinical Psychology, 68, 728733. 
89. Werch, C.E., Carlson, J.M., Pappas, D.M. \& DiClemente, C.C. (1996). Brief nurse consultations for preventing alcohol use among urban school youth. Journal of School Health, 66, 335-338.
90. Foxcroft, D.R., Lister-Sharp, D. \& Lowe, G. (1997) Alcohol misuse prevention for young people: a systematic review reveals methodological concerns and lack of reliable evidence of effectiveness. Addiction, 92, 531-537. 